DOE/NV/25946--109

\title{
FLASH X-RAY DIFFRACTION SYSTEM FOR ULTRAFAST TEMPERATURE AND PHASE TRANSITION MEASUREMENTS
}

\author{
Dane V. Morgan, Don R. Macy, Michael J. Madlener, \\ and Jiaming G. Morgan \\ National Security Technologies, LLC, 182 East Gate Drive, \\ Los Alamos, NM 87544 USA
}

A novel ultrafast diagnostic for determining bulk temperature and phase transitions for polycrystalline metal objects has been developed. The diagnostic consists of a 38-stage Marx bank with a cable-coupled X-ray diode that produces a 35-ns pulse of mostly $0.71 \AA$ monochromatic X rays, and a P-43 fluor coupled to a cooled charge-coupled device camera by a coherent fiber-optic bundle for detection of scattered $\mathrm{X}$ rays. The $\mathrm{X}$-ray beam is collimated to a $1^{\circ}$ divergence in the scattering plane with the combination of a $1.5-\mathrm{mm}$ tungsten pinhole and a $1.5-\mathrm{mm}$ diameter molybdenum anode. The $\mathrm{X}$-ray diode, in a needle-and-washer configuration, is heavily shielded in all directions other than the collimated beam. The $\mathrm{X}$-ray diode has a sealed reentrant system, which allows the $\mathrm{X}$ rays to be produced inside a vacuum containment vessel, close to the sample under study.

The direct correlation between the solid-state structure and the coherent X-ray diffraction pattern from a metal surface allows an unequivocal determination of a phase transition. This correlation has been tested in the laboratory with samples of indium and tin. For both metals, diffraction lines were observed at temperatures just below the melt temperature, along with background consisting of Compton scattering and sample fluorescence. Upon melt, the diffraction lines were observed to disappear; however, the background from Compton scattering and sample fluorescence remained.

Flash X-ray diffraction also enables direct ultrafast measurements of the bulk temperature of the sample under study. According to the Debye-Waller theory, the diffracted line intensity reduces as the temperature of the sample increases. ${ }^{1}$ The amplitude of the reduced diffracted signal also depends on the Debye temperature of the sample, the scattering angle of the diffracted $\mathrm{X}$ rays, and the X-ray wavelength. The feasibility of using the Debye-Waller theory for flash X-ray diffraction measurements of the bulk temperature is currently being studied.

1. C. Kittel, Introduction to Solid State Physics, $6^{\text {th }}$ edition, New York: John Wiley and Sons, 1986, pp. 603-605.

This work was done by National Security Technologies, LLC, under Contract No. DE-AC52-06NA25946 with the U.S. Department of Energy. 